\title{
Diagnóstico precoz de caries dental utilizando fluorescencia laser: Parte I
}

Precocious diagnose of dental decay using laser fluorescence: Part I

\begin{abstract}
Resumen
La dificultad para el diagnostico de las lesiones cariosas tempranas en dientes temporales nos ha estimulado a desarrollar este trabajo y mejorar la exactitud del diagnostico con el uso de la florescencia inducida por luz láser. Sabemos que la superficie oclusal es considerada la zona más vulnerable al desarrollo de lesiones cariosas, estando esta alta susceptibilidad relacionada directamente con su morfología llena de clevaciones y depresiones que favorccen la retención de microorganismos y restos alimenticios. Comúnmente el diagnostico de una lesión cariosa oclusal se efectúa mediante una combinación de técnicas que incluyen el examen clínico con el uso del explorador y por medio de las radiografías. El propósito de esta investigación es determinar la efectividad del examen visual y radiográfico en el diagnostico de lesiones cariosas incipientes y compararlas con los resultados arrojados de la técnica con fluorescencia láser.
\end{abstract}

\section{Abstract}

The difficulty for the diagnosis of carieo lessions in temporal teeth has made this work improve in a closer diagnosis using the Fluorescence of the Laser. As we know the most sensitive part for caries is the Oclusal surface, because of the characteristic morphology that this piece presents, make the micro organisms and rest of food to stay. Normally the caries diagnosis on the oclusal surface is determinated by a combination of different techniques that includes the intra oral exam with the use of an explorer and radiographies. The purpose of this research is to determinate the efficiency of the Visual and radiographics exam in the diagnosis of incipient caries lesion and compare them with the results found with the fluorescence laser techrique.

\section{Introduccion}

La caries dental es una de las enfermedades de mayor prevalencia en el hombre. Existe una gran cantidad de investigaciones al respecto, por lo tanto cada día se conocen mas sobre su proceso y diagnóstico tempranao, lo cual permite revertirla cuando es tratada a tiempo.

El diagnóstico de una lesión de caries se hace generalmente mediante una combinación de técnicas que incluyen el examen visual con el uso del explorador y las radiografías de aleta de mordida. El uso del explorador ha sido sumamente cuestionado debido a las afirmaciones de Loesche ${ }^{1}$ en 1972 que el uso del explorador puede transmitir el Streptococcus mutans de una fisura contaminada a otra fisura supuestamente sana. Thylstrup y Yassin ${ }^{2}$ en 1995 opinan que la presión ejercida con el explorador durante el examen puede causar daño en aquellas fisuras y lesiones incipientes con posibilidad de remineralización, favoreciendo de esta modo la progresión de la caries. Según Lussi ${ }^{3}$ (1991) y Penning 4 (1992) el uso del explorador no mejora la precisión del diagnóstico de las lesiones cariosas en fosas y fisuras, con respecto a la inspección visual. Housseiny y $\mathrm{col}^{5}$ (2001) y Heinrich y $\operatorname{col}^{6}$ (2002) determinan que el examen visual presenta una baja sensibilidad y una alta espe-

\author{
Celso Guillén Borda ${ }^{1 *}$; Silvia Chein Vil- \\ lacampa ${ }^{2 *}$; Segundo Perales Zamora ${ }^{3 *}$; \\ María Veniosilla Huasupoma ${ }^{4}$; Jorge \\ Villavicencio Gastelút ${ }^{*}$; Carlos Rivas \\ Butrón( $(\uparrow)^{6^{*}}$; Sergio Alvarado Menacho ${ }^{7 *} y$ \\ Anionio Guillén Astete ${ }^{8}$
}

\footnotetext{
1,3 Departamento Académico de Estomatología Pediátrica

2 Departamento Académico de Estomatología Biosocial

4,7 Departamento Académico de Estomatología Rehabilitadota

5,6 Departamento Académico de Estomatologia Medico Quirúrgico

8 Consulta privada, av. Petit Thaurs N*2871, Lima, Perú

* Facultad de Odontología, Universidad Nacional Mayor de San Marcos, Lima, Perú E-mail: cguillenb@unmsm.edu.pe
}

Palabras clave: Caries, dientes temporales, fluorescencia

Key words: Caries, temporary tceth, fluorescence cificidad sobre el examen radiográfico. En la practica clínica se observamos lo dificultoso del diagnóstico de las de las lesiones incipientes en fosas y fisuras a través de las radiografías. En los últimos años se ha incrementado la cantidad de herramientas de alta tecnología ${ }^{7-10}$ para un diagnostico de caries mas preciso debido a la dificultad para diagnosticar correctamente las lesiones tempranas de caries. Dentro de estas nuevas técnicas de diagnóstico, parecen ser efectivas las que se basan en la fluorescencia inducida con Láser. Hibst y Gall ${ }^{11}$ en 1998, Kavo Diagnodent ${ }^{3}$, desarrollaron el equipo láser portátil "DIAGNOdent" como una alternativa al examen vi- 
sual y radiográfico de las lesiones de caries en superficies lisas y oclusales. $\mathrm{E}$ l instrumento mide la cantidad de luz fluorescente irradiada, del tejido dental desmineralizado; como resultado de la excitación inducida por un láser diodo (Clase 2) que emite una longitud de onda de $655 \mathrm{~nm}$ con una potencia de $1 \mathrm{~mW}$. Según Sundström12 dice que el principio se basa en que los cambios inducidos en la estructura dentaria, por el proceso de caries, lleva a un incremento en la fluorescencia cuando se aplica longitudes de onda específicas.

El tipo de láser utilizado en el DIAGNOdent es un diodo semiconductor que ilumina la superficie dental, a través de una sonda flexible, con una luz láser roja intermitente, que penetra varios milímetros dentro de la estructura dentaria. Una parte de la luz es absorbida por los componentes orgánicos e inorgánicos de la estructura dental, mientras que otra parte de esta luz es reemitida como fluorescencia, dentro del espectro infrarrojo, hacia el dispositivo a través de nueve fibras óplicas colocadas alrededor de una fibra óptica central, siendo la información analizada y cuantificada por los fotodiodos que se encuentran en el interior del equipo.

\section{Materiales y Métodos}

La población programada para este estudio de carácter Experimental es de 600 molares superiores e inferiores deciduas de niños comprendidos dentro del grupo etáreo de 6 a 8 años. Los datos para el presente estudio se están recolectando en el I Iospital del Niño, Clínicas Periféricas y en la Clínica Montefiori. Cada uno de ellas se encuentran como par de molares superiores e inferiores (Cuadro 1), que constituyen la muestra.

El método a emplear en este trabajo, es el Clínico-Radiográfico mediante el siguiente protocolo: Examen clínico visual, examen radiográfico y examen por Fluorescencia con Láser. Luego de realizar una profilaxis al niño, se aísla con rollos de algodón, se observan las piezas molares deciduas por oclusal, mesial, distal, vestibular y palatino con espejo y explorador. Posteriormente se toman radiografías de mordida en ambos sectores y se procede a la detección de caries mediante la Fluorescencia Laser (DIAGNOdent), siguiendo el protocolo indicado por el fabricante.
Cuadro 1: Primer informe de la población programada por numero de pares de molares superiores e inferiores según ubicación de la sede de investigación

\begin{tabular}{|c|c|c|c|c|c|c|c|c|c|c|}
\hline \multirow{3}{*}{$\begin{array}{l}\text { Jefe de } \\
\text { Investiga- } \\
\text { ción }\end{array}$} & \multirow{2}{*}{\multicolumn{2}{|c|}{$\begin{array}{c}\text { Población } \\
\text { programada }\end{array}$}} & \multirow{3}{*}{\begin{tabular}{|c|} 
Nú- \\
mero \\
de \\
pares \\
de $\mathrm{M}$.
\end{tabular}} & \multirow{3}{*}{\begin{tabular}{|c|} 
Nú- \\
mero \\
de \\
Aten- \\
didas
\end{tabular}} & \multirow{3}{*}{$\begin{array}{c}\text { Por- } \\
\text { cen- } \\
\text { taje } \\
\text { de } \\
\text { Aten- } \\
\text { didos }\end{array}$} & \multirow{3}{*}{\begin{tabular}{|c|} 
Nú- \\
mero \\
de \\
pares \\
de \\
M.S.
\end{tabular}} & \multirow{3}{*}{\begin{tabular}{|c|} 
Por- \\
cen- \\
taje \\
M.S. e \\
l aten- \\
didas
\end{tabular}} & \multicolumn{2}{|c|}{ Sexo } & \multirow[t]{3}{*}{ Edad } \\
\hline & & & & & & & & \multirow[t]{2}{*}{$\mathrm{M}$} & \multirow[t]{2}{*}{$\mathrm{F}$} & \\
\hline & \begin{tabular}{|c|}
$N^{o}$ \\
de \\
niños
\end{tabular} & $\begin{array}{c}N^{o} \text { de } \\
\text { MS.e } \\
\text { I por } \\
\text { niños }\end{array}$ & & & & & & & & \\
\hline $\begin{array}{l}\text { Hospital del } \\
\text { Niño }\end{array}$ & 50 & 4 & 200 & 23 & $46 \%$ & 86 & $46 \%$ & 14 & 9 & $6-8$ \\
\hline $\begin{array}{l}\text { Clínica } \\
\text { Periférica }\end{array}$ & 50 & 4 & 200 & 18 & $36 \%$ & 65 & $32.5 \%$ & 8 & 10 & $6-8$ \\
\hline $\begin{array}{l}\text { Clínica } \\
\text { Montifiori }\end{array}$ & 50 & 4 & 200 & 19 & $38 \%$ & 69 & $34.5 \%$ & 8 & 11 & $6-8$ \\
\hline Total & 150 & 4 & 600 & 60 & $40 \%$ & 220 & $33.3 \%$ & 30 & 30 & $6-8$ \\
\hline
\end{tabular}

Leyenda:

$\mathrm{M}=$ Molares

$S$ - Superior

$I=$ Inferior

\section{Resultados}

Se pudo observar que el examen clínico visual, no es un método fiable para la detección de caries dental en fosas y fisuras. Las caries interproximales están siendo detectadas con mayor facilidad mediante la toma de radiografías. El DIAGNOdent esta detectando eficazmente la placa bacteriana, depósitos orgánicos, sarro y restos alimenticios.

La lectura, para cstablecer la efectividad del láser en el diagnóstico de las

lesiones cariosas, implica la necesidad de tener un alto conocimiento de anatomía dentaria para poder interpretar de la mejor manera los datos arrojados por el DIAGNOdent durante su barrido. La fluorescencia laser pudo detectar caries dentinaria, establecida por la localización las lesiones cariosas y cambios en la anatomía dentinaria.

A continuación, presentamos valores preliminares correspondientes a la primera fase de este estudio, 12 de 24 meses (cuadros 2-5).

Cuadro 2: Distribución de molares examinados mediante las Técnicas Visual, Radiográfica y Láser. Hospital del Niño.

\begin{tabular}{l|rr|rr|rr}
\hline Técnica del Diagnóstico & \multicolumn{2}{|c|}{ Visual } & \multicolumn{2}{c|}{ Radiográfico } & \multicolumn{2}{c}{ Laser } \\
\hline Condición & \multicolumn{1}{|c|}{$\mathrm{N}^{0}$} & $\%$ & $\mathrm{~N}^{0}$ & $\%$ & $N^{0}$ & $\%$ \\
\hline Sano & 47 & $54 \%$ & 57 & $66 \%$ & 20 & $23 \%$ \\
Caries & 39 & $46 \%$ & 29 & $34 \%$ & 66 & $77 \%$ \\
Total & 89 & $100 \%$ & 86 & $100 \%$ & 86 & $\mathbf{1 0 0 \%}$ \\
\hline
\end{tabular}

Cuadro 3: Distribución de molares examinados mediante las Técnicas Visual, Radiográfica y Láser. Clínica Periférica

\begin{tabular}{l|rr|rr|rr}
\hline Técnica del Diagnóstico & \multicolumn{2}{|c|}{ Visual } & \multicolumn{2}{c|}{ Radiográfico } & \multicolumn{2}{c}{ Laser } \\
\hline Condición & $\mathrm{N}^{0}$ & $\%$ & $\mathrm{~N}^{0}$ & $\%$ & $\mathrm{~N}^{\circ}$ & $\%$ \\
\hline Sano & 28 & $58 \%$ & 40 & $61 \%$ & 18 & $27 \%$ \\
Caries & 27 & $42 \%$ & 25 & $39 \%$ & 47 & $73 \%$ \\
Total & 65 & $100 \%$ & 65 & $100 \%$ & 65 & $100 \%$ \\
\hline
\end{tabular}


Cuadro 4: Distribución de molares examinados mediante las Técnicas Visual, Radiográfica y Láser. Clínica Montefiori

\begin{tabular}{|c|c|c|c|c|c|c|}
\hline Técnica del Diagnóstico & \multicolumn{2}{|c|}{ Visual } & \multicolumn{2}{|c|}{ Radiográfico } & \multicolumn{2}{|c|}{ Laser } \\
\hline Condición & $\mathrm{N}^{\circ}$ & $\%$ & $\mathrm{~N}^{\mathrm{o}}$ & $\%$ & $\mathrm{~N}^{\circ}$ & $\%$ \\
\hline Sano & 28 & $40 \%$ & 43 & $62 \%$ & 26 & $37 \%$ \\
\hline Caries & 41 & $60 \%$ & 26 & $38 \%$ & 43 & $63 \%$ \\
\hline Total & 69 & $100 \%$ & 69 & $100 \%$ & 69 & $100 \%$ \\
\hline
\end{tabular}

Cuadro 5: Total de molares examinadas mediante la Técnica Visual, Radiográfica y Láser (3 sedes).

\begin{tabular}{l|cr|cc|cr}
\hline Técnica del Diagnóstico & \multicolumn{2}{|c|}{ Visual } & \multicolumn{2}{c|}{ Radiográfico } & \multicolumn{2}{c}{ Laser } \\
\hline Condición & $\mathrm{N}^{\circ}$ & $\%$ & $\mathrm{~N}^{\circ}$ & $\%$ & $\mathrm{~N}^{\circ}$ & $\%$ \\
\hline Sano & 113 & $51 \%$ & 140 & $63 \%$ & 64 & $29 \%$ \\
Caries & 107 & $49 \%$ & 80 & $37 \%$ & 156 & $71 \%$ \\
Total & 220 & $100 \%$ & 220 & $100 \%$ & 220 & $100 \%$ \\
\hline
\end{tabular}

\section{Discusión}

El uso del Equipo Láser puede leer a una profundidad de $2 \mathrm{~mm}$ en el interior de la pieza dental, detectando "fluorescencias" en cualquier zona en que se aplique. Es capaz de detectar una elevada fluorescencia natural en la pieza dental, la placa bacteriana y los depósitos orgánicos, resinas compuestas y márgenes desmineralizados, sarro, dentina hipocalcificada y cariada. Presenta una alta sensibilidad y baja especificidad. Por ello permite Ilevar a cabo un diagnóstico precoz de la caries dental y tratar a tiempo las lesiones cariosas.

El DIGNOdent, nos permite reconocer y diagnosticar precozmente los cambios patológicos en los tejidos dentarios. La fluorescencia producida por el Láser permite localizar rápidamente las lesiones iniciales desmineralizadas. En el futuro el diagnóstico de la caries dental con fluorescencia laser asegura una mejor conservación de los tejidos dentarios.

\section{Conclusiones}

Siendo la caries dental una de las enfermedades de mayor prevalencia del sistema estomatognático, afectando a un $95 \%$ de la población, sobre todo a los niños, y cuyo diagnóstico se torna dificil principalmente en sus etapas iniciales, la fluorescencia con Láser se presenta como una alternativa interesante y más exacta que los otros métodos de diagnóstico:

- Se mejora la exactitud en el diagnostico de la caries incipiente

- Capacidad de monitorizar la caries dental

- El Paciente "ve y escucha” el diagnóstico de caries

- La terapia es preventiva

- Conservación de la salud de los tejidos dentarios

\section{Referencias}

1. Loesche WJ, Hockett RN, Syed SA. The predominant cultivable flora of tooth surface plaque removed from institutionalized subjects. Arch Oral Biol. 1972 Sep;17(9):1311-1325.

2. Thylstrup A., Yassin, L.: Light microscope study of the effect of probing on the occlusal surfaces. Caries Res $1995 ; 21: 368 \neg 74$.

3. Lussi A. Validity of a diagnostic and treatment decision of fissure caries. Caries Res 1991; 25:296-303.

4. Penning C, Van Amerogen JP, Seef $\mathrm{RE}$, ten Cate JM. Validity of probing for fissure caries diagnosis. Caries Res 1992; 26:445-9.

5. Housseiny AA, Jamjoum H. Evaluation of visual, explorer and a laser device for detection of early occlusal caries. J Clin Pediatr Dent 2001; 26(1):41-8.

6. Heinrich-Weltzien R, Weerheijm KI, Kühnisch J, Oehme T, Stösser L, Clinical evaluation of visual, radiographic and a laser fluorescence methods for detection of occlusal caries. J Dent Child 2002; 69(2):127-32

7. Tam LE, McComb D. Diagnosis of occlusal caries: Part II. Recent diagnostic technologies. J Can Dent Assoc 2001; $67(8): 459-63$

8. Kavo DIAGNO dent. Diagnostico de caries y como utilizar el sistema DIAGNO dent (en CD - ROM). Biberach: Kavo Dental Excellence; 2002

9. Shi $X-Q$, Welander U, AngmarMansson $B$. Occlusal caries detection with Kavo DIAGNO dent and radiography: An in vitro comparison. Caries Res 2000; $34: 151-8$

10. Anttonen V, Seppa 1, Hausen H. Clinical study of the use of laser fluorescente device DIAGNO dent of detection of occlusal caries in children. Caries Res 2003; 37(1): $17-23$

11. Hibst R, Gall R. Development of a diode laser-based fluorescence caries detector [abstract]. Caries Res 1998; 32:294

12. Sundström F, Fredriksson K, Montán S, Hafström-Björkamn U, Ström J. Laserinduced fluorescente from sound and carious tooth substance. Spectroscopic studies. Swed Dent J 1985; 9:71-80. 